AUTHOR'S POST PRINT (Romeo Colour: Green)

Acta Astronautica (ISSN: 0094-5765) Vol. 47, Nos. 2-9, pp. 325-334, 2000.

DOI: $10.1016 / \mathrm{S} 0094-5765(00) 00074-6$

Publisher version available at

http://www.sciencedirect.com/science/article/pii/S0094576500000746

\title{
FLIGHT RESULTS ON MARANGONI FLOW INSTABILITY IN LIQUID BRIDGES
}

\author{
R. MONTI, R. SAVINO, M. LAPPA* \\ Università degli Studi di Napoli "Federico II" \\ Dipartimento di Scienza e Ingegneria dello Spazio "Luigi G. Napolitano" \\ P.le V.Tecchio 80, 80125 Napoli (Italy) \\ *current e-mail address: marcello.lappa@strath.ac.uk \\ L. CAROTENUTO, D. CASTAGNOLO, R. FORTEZZA \\ MARS Center, Via Comunale Tavernola, 80144 Napoli (Italy)
}

\section{Abstract}

This paper reports on the results of the experiment PULSAR (Pulsating and Rotating Instabilities in Oscillatory Marangoni Flows), performed on the MAXUS 3 Sounding Rocket launched last November from the Swedish base in Kiruna. Aim of the experiment was the study of the oscillatory Marangoni convection in a cylindrical liquid bridge of silicone oil with kinematic viscosity of $5 \mathrm{cSt}$. The experiment was motivated by preliminary on-ground numerical simulations and microscale experimental studies, that have pointed out that the oscillatory Marangoni instability appears at the beginning in the form of a pulsating regime, caused by a hydro-thermal standing wave, and then it turns to a rotating regime, caused by a traveling wave. The height of the bridge was equal to the disk diameter $(20 \mathrm{~mm})$, and the imposed temperature difference was $15 \mathrm{~K}$ during the first $460 \mathrm{~s}$ and $20 \mathrm{~K}$ in the second part of the experiment, until the end of the microgravity period.

The analysis of the temperature profiles, measured by thermocouples located near the disks at the same radial and axial coordinate but at different azimuthal coordinates (shifted at $90^{\circ}$ ), and the surface temperature distribution, measured by an infrared thermocamera, show that a pulsating and a mixed pulsating-rotating regimes have been established during the experiment.

Unfortunately during the flight the accelerations level caused by two centrifuges with some biological samples in an adjacent module were above the expected values, so that disturbing g-jitter were encountered at different times during the microgravity mission. The effecst are clearly visible and the numerical simulations had to make different assumptions to correlate the experimental results

\section{$\underline{1 . \text { Introduction }}$}

The existence of a critical Marangoni number for the onset of the time-dependent asymmetric flow in high Prandtl number fluids has been established two decades ago [1]. Experiments show that for a liquid bridge of a given geometry, in the presence of sufficiently small temperature differences, the Marangoni convection is characterized by a steady, axisymmetric, toroidal flow pattern driven by the surface tension imbalance. When the temperature difference exceeds a critical value, depending on the geometry, on the liquid properties and on the boundary conditions, the flow experiences a transition to an oscillatory and three-dimensional pattern. A number of experimental works on the subject have been performed, on ground and in microgravity conditions, by different investigators [2-10].

From the theoretical/numerical point of view oscillatory thermocapillary flows in liquid bridges have been investigated by the hydrodynamic stability theory $[11,12]$. For high Prandtl numbers this theory predicts that the three dimensional supercritical state after 
the onset of instability can be interpreted as a superposition of two counter-propagating waves, characterized by an axial and an azimuthal component, with a front wave forming a certain angle with the axis of the bridge. The superposition of two waves with equal amplitude should result in a "standing wave", with the minimum and maximum disturbances at fixed azimuthal positions; while the superposition of waves with different amplitude should give rise to a "traveling wave", with the minimum and maximum disturbances traveling in azimuthal direction. Nothing can be predicted by linear stability analyses about the amplitude of these disturbances. From the experimental point of view, the traveling wave model was observed by different investigators on ground and in microgravity [2-5]. The standing wave model, instead, has been observed more recently in ground-based experiments $[7,8]$ and during a Spacelab experiment [9]. On the other hand, the development of supercomputers and of efficient numerical methods allowed direct numerical solutions of the three-dimensional and time-dependent Navier Stokes equations. Savino and Monti [13] and Monti, Savino, Lappa [14] have shown that the standing wave and the traveling wave models correspond to two consecutive transitions of the Marangoni flow. To confirm these numerical results the experiment PULSAR has been performed onboard the MAXUS 3 Sounding Rocket launched last November from the Swedish base in Kiruna. A previous paper ([15] reported on the activities in preparation of the experiment, consisting in extensive numerical simulations, complemented by laboratory experiments performed with a microscale apparatus.

Aim of the PULSAR experiment was to study the flow and temperature distributions of the oscillatory Marangoni convection after the onset of instability. For this purpose the Marangoni flow in a cylindrical liquid bridge of a $5 \mathrm{cSt}$ silicone oil was investigated. The attention has been focused on the three dimensional structure of the flow that appears once the temperature difference between the disks supporting the bridge exceeds a certain critical value.

\section{Description of the flight hardware and of the diagnostic}

The experimental model is an improved version of the TEM 06-4 that has already been used during previous experiments on Texus and Maxus missions.

A pressurized test cell contains the liquid bridge (Silicone oil) between two disks formed after the microgravity conditions are established. The liquid reservoir, closed during the acceleration phase, is opened by an electromechanical device and the exact quantity of liquid needed to form a cylindrical liquid bridge is pushed between the two copper disks while the disks are moving to reach the prescribed distance. The active cooling and heating system is designed to reduce the heat transfer from the oil to the ambient. The system, based on two Peltier elements, allows to control the temperature ramps of the two disks imposing symmetric temperature profiles with respect to the initial temperature; the supports are equipped with thermocouples in order to measure and control the temperature.

Additional thin thermocouples placed at four fixed points having the same radial coordinates, but at angles of 90 degree (see Fig. 1), are used for the analysis of the liquid temperature inside the liquid bridges during the experimental phases (the thermocouples protrude in the bridge in axial direction; they are hosted in apposite holes in the disks sustaining the bridge when the two disks are in launch configuration), as illustrated in Fig. 1a.

The liquid motion is visualized using tracers illuminated by a laser diode forming a light sheet passing through the symmetry axis. The images are taken by a CCD camera and transmitted to ground.

The experiment has been fully controlled in Telescience operative mode directly from MARS Center (Italy) control room by using four dedicated ISDN lines, while the rocket has been launched from Kiruna (Sweden). 
The laser beam was oriented orthogonal to the main optical path of the first CCD. The laser used is He-Ne with a wavelength of 635 $\mathrm{nm}$. The images detect the tracers motion contained in the cylinder meridian plane of the liquid bridge.

The sketch of the Pulsar Experiment plate arrangement is illustrated in Fig. 2. The two IR mirrors located on the left and on the right of the liquid bridge are used to observe simultaneously two opposite surface sides. This set-up allows the detection of the surface hot and cold spots and the analysis of both pulsating and rotating oscillation regimes The IR camera used is an AGEMA 550 able to generate $15 \mathrm{fps}$ transmitted to ground for the experiment control. At the same time the camera was able to store onboard digital images at 1 fps on a flash memory.

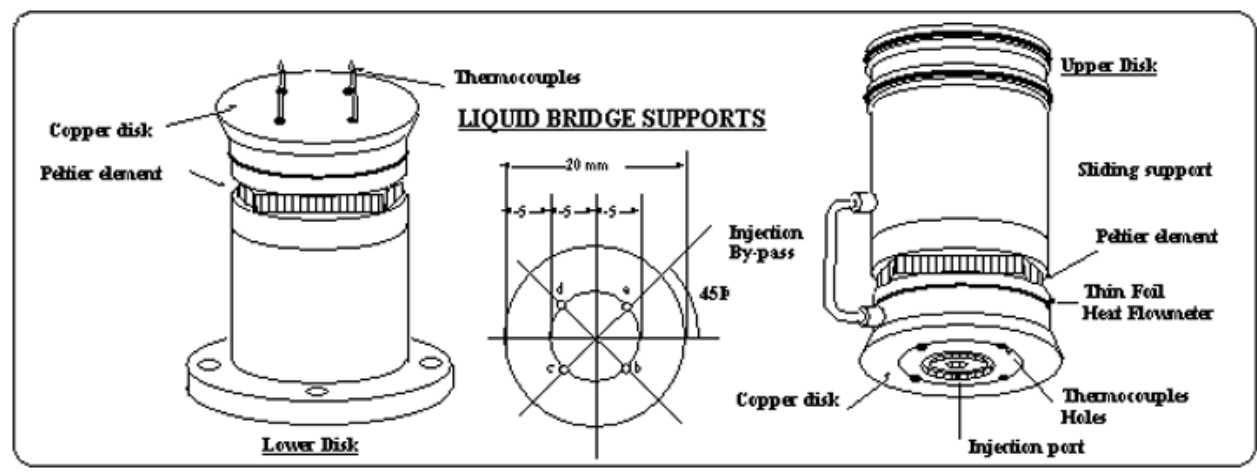

Fig. 1 - Sketch of the liquid bridge support and thermocouple location

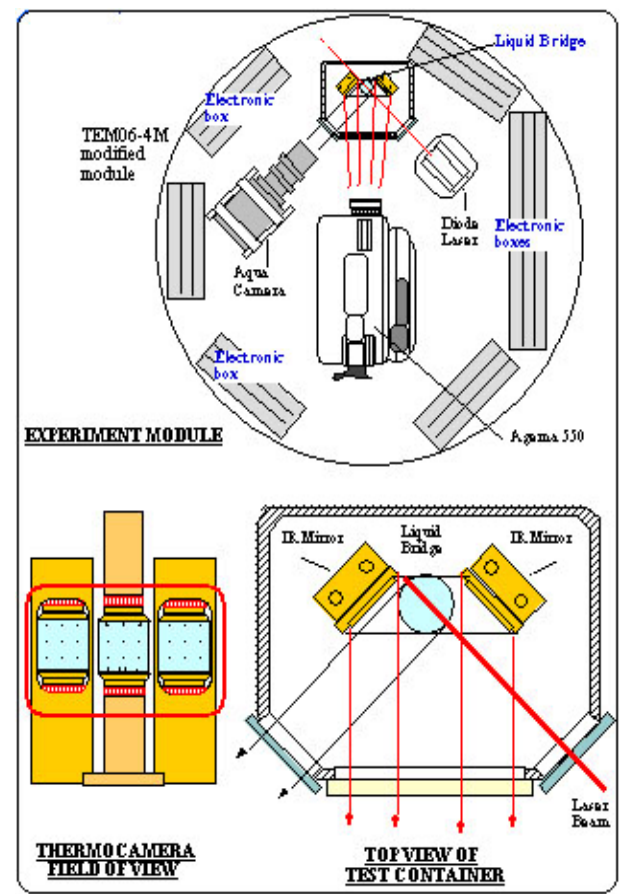

Fig. 2 - Sketch of PULSAR Experimental Plate

\section{Results of the PULSAR experiment}

A post flight analysis of the data and video recorded during the experiment onboard the
MAXUS 3 sounding rocket mission was carried out.

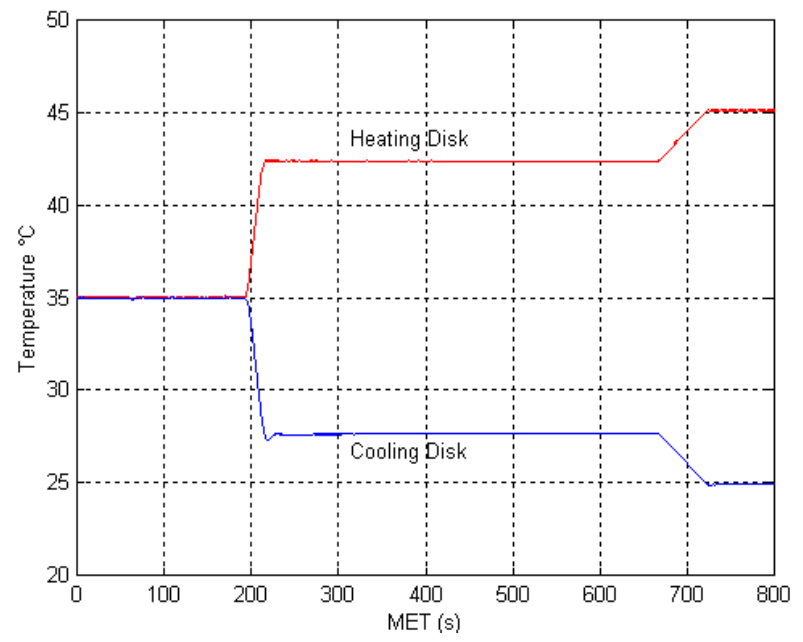

Fig 3. History of the setting temperatures during 800 seconds of the Pulsar experiment. The upper line represents the setting temperature on the heating disk, the lower one on the cooling disk.

The experiment chamber has been thermostated to $35{ }^{\circ} \mathrm{C}$ prior to lift off. The temperature evolution on the supporting disks during the first 800 seconds from the rocket launch is shown in Fig.3. The microgravity period began after 100 seconds; the bridge was formed and the thermocamera was set 
during the successive 90 seconds. The height of the bridge was equal to the disk diameter $(20 \mathrm{~mm})$. An initial temperature difference of $15^{\circ} \mathrm{C}$ was established by means of symmetric ramp $\left( \pm 0.4^{\circ} \mathrm{C} / \mathrm{s}\right)$ and held constant during the first 461 seconds from MET=219 to MET $=680$.

Then, the temperature difference was raised up to $20^{\circ} \mathrm{C}\left( \pm 0.05^{\circ} \mathrm{C} / \mathrm{s}\right)$ in the second part of the experiment and held constant for 100 seconds, until the end of the microgravity period.

Because of the presence of a centrifuge, placed in a biological module adjacent to TEM 06-4, vibrations have been observed both in video and in thermocamera images. The rocket was forced to roll along its longitudinal axis, and some g-peaks occurred at some moments as consequence of the acceleration. The influence of these vibration on the dynamics of the flow organization inside the bridge is discussed in the following paragraphs.

Fig. 4 reports the time profiles of the roll angular velocity $\left(\omega_{\mathrm{x}}\right)$ and of the differences between the signals of the thermocouples shifted at $180^{\circ}\left(\mathrm{T}_{\mathrm{z1}}-\mathrm{T}_{\mathrm{z} 3} ; \mathrm{T}_{\mathrm{z} 2}-\mathrm{T}_{\mathrm{z} 4}\right)$.

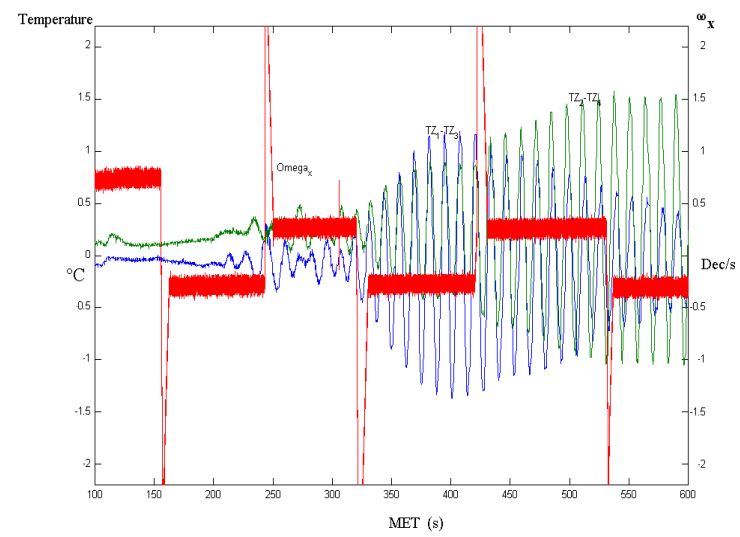

Fig.4 Time plots of the signal difference between thermocouples shifted at $180^{\circ}$ on the right side and of the longitudinal angular velocity on the left side.

It is evident that the centrifuge was activated at different times causing strong angular accelerations. For instance at MET 421s, there is an angular acceleration of about 0.106 $\mathrm{rad} / \mathrm{s}^{2}$ for 0.6 seconds and a successive angular deceleration of about $0.0072 \mathrm{rad} / \mathrm{s}^{2}$ during 8.6 seconds.
Considering that the distance between the experiment location and the rocket axis was $19 \mathrm{~cm}$, these two values correspond to tangential accelerations $\approx 2.0610^{-3}$ and $\approx 1.4$ $10^{-4} \mathrm{~g} / \mathrm{g}_{0}$ respectively.

The corresponding centripetal acceleration was in turn smaller than the tangential ones. $\approx 2.5510^{-5} \mathrm{~g} / \mathrm{g}_{0}$.

The method used for the present numerical simulations is described elsewhere (see e.g. [16] where the complete numerical procedure is presented in detail).

Figs. 5 and 6 report a comparison between numerical and experimental results. The yaxis shows the temperatures of two thermocouples (or probes in the numerical simulation) azimuthally shifted at $90^{\circ}$. Fig. 5 is relative to the thermocouples near the hot disk, Fig. 6 to the thermocouples near the cold one. The numerical simulation (Figs. 5a and 6a) shows that a standing wave is established at the onset of the periodic oscillations and lasts for long time.

The difference between a standing and a traveling wave consists in the fact that in the first case, for $m=1$, only two values of the phase shift are allowed, while in the second case the possible values of the phases are not discrete. In particular, the pulsating regime is characterized by signals of $\mathrm{T}_{\mathrm{Z} 1}-\mathrm{T}_{\mathrm{Z} 3}$ and $\mathrm{T}_{\mathrm{Z} 2}-\mathrm{T}_{\mathrm{Z} 4}$ in phase.

This behaviour is directly related to the behaviour of the azimuthal temperature spots induced by the instability. Each of these spots has, in the case $m=1$, an angular extension of 180 degree so that two thermocouples will be located on the hot spot, and two on the cold one.

In the standing wave regime these spots have fixed azimuthal position and "pulsate" in time.

Thus the phase jump is 0 if the considered points are placed on the same azimuthal spot, $\pi$ if the two points belong to two different spots.

In the rotating regime the possible values of the phases are not discrete since the azimuthal spots travel continuosly in azimuthal direction.

Moreover in a standing wave regime the temperature signals have not the same 
amplitude (typically two signals have amplitude greater that that of the other two) whereas in a rotating regime all the signals show the same amplitude.

In Figs. 5a and $6 \mathrm{a}$ it is evident that there is no phase shift between the temperature signals measured by two thermocouples at 90 degree. The phase shift is instead $\pi$ when the two considered thermocouples have an azimuthal shift of 180 degree. Moreover the signals do not have the same amplitude.

The numerical simulations show that the pulsating regime is followed by a rotating regime after a time longer than the available microgravity time.

The instability regime (pulsating or rotating) can be identified by looking at the differences of the phase of the temperature profiles as described above or, from a fluid-dynamic point of view, by looking at the behaviour of the tracer particles in meridian planes having different azimuthal coordinate.

The pulsating regime is characterized in fact by the existence of a "fixed plane" called the "pulsation" plane; the axis of the deformed Marangoni toroidal convection roll inclined with respect to the zone axis due to the onset of instability, oscillates in this plane forward and backward. Observations in direction orthogonal to this plane should show the characteristic pendulum motion of the branching streamlines that divides the two asymmetrical convection cells in the meridian plane. Observations in other planes should show instead a symmetric flow.

In the rotating regime the flow behaviour should be the same in each meridian plane due to the fact that the inclined axis of the deformed Marangoni three-dimensional convection roll rotates in azimuthal direction. This rotation gives rise to an asymmetric flow field in each meridian plane.

The experimental signals (Figs. 5b and 6b) seem to show two different phases. During a first time range (say $300<\mathrm{MET}<660$ ) a standing wave is established; in a second time range (say $420<\mathrm{MET}<500$ ) a mixed regime is established.

Due to the behaviour of the temperature signals (the values of the differences of phase are discrete, but there is a variation of their amplitude in time) it can be argued that the "mixed" regime was caused by the relative rotation of the pulsating plane around the bridge axis, probably induced by the strong angular acceleration occurred at MET $=421$ seconds, that caused a possible relative rotation of the standing wave.

If the regime is pulsating, but the azimuthal position of the "pulsation plane" changes in time, the relative position of the thermocouples and of the temperature spots should in fact change in time giving rise to a variation of the amplitudes of the signals not associate to a change of their phases.

Looking at the temperatures of the thermocouples near the hot disk (Fig. 5b) the amplitude of a temperature signal decreases whereas the amplitude of the other signal increases.

This behaviour has been numerically simulated with a rotation of the pulsating plane around the bridge axis with an angular speed of $0.15 \mathrm{deg} / \mathrm{s}$ (Fig. 5c).

Figs. 6 correspond to the temperatures of two thermocouples at $90^{\circ}$ near the cold disk.

The angular phase difference between the two temperature signals is 0 in the first period and $\pi$ after that the rotation of the pulsating plane has occurred (no intermediate values are observed).

Fig. 6c shows that, considering a relative rotation between the temperature sensors and the bridge axis, the numerical temperature signals are in sufficient agreement with the experimental ones (Fig. 6b).

These results can also be confirmed by looking at both video and thermocamera images captured at some relevant moments. Figure 7 reports a sequence of images of the thermocamera equipment during one half of the oscillation period prior to the MET $=421$ and the corresponding numerical results.

The images are obtained by black and white reproduction of the coloured thermocamera images that represent the temperature disturbances on the liquid bridge surface. 


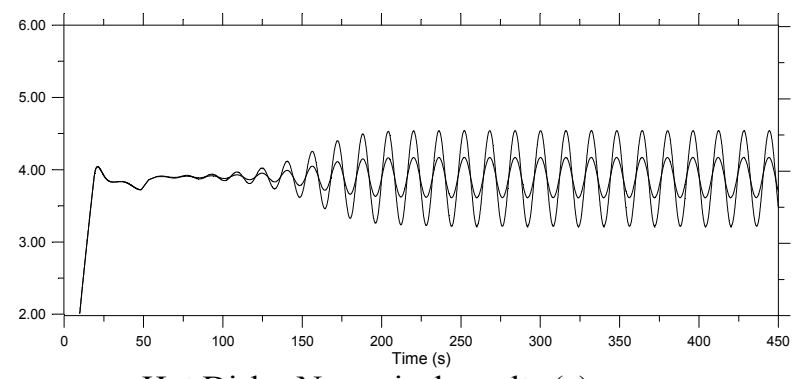

Hot Disk - Numerical results (a)

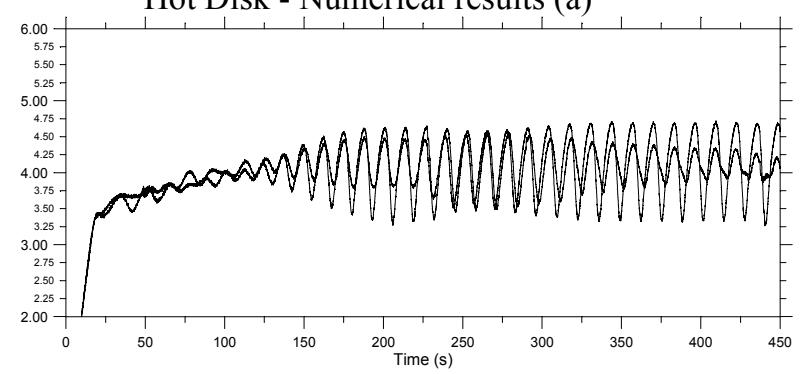

Hot Disk - Experimental results (b)

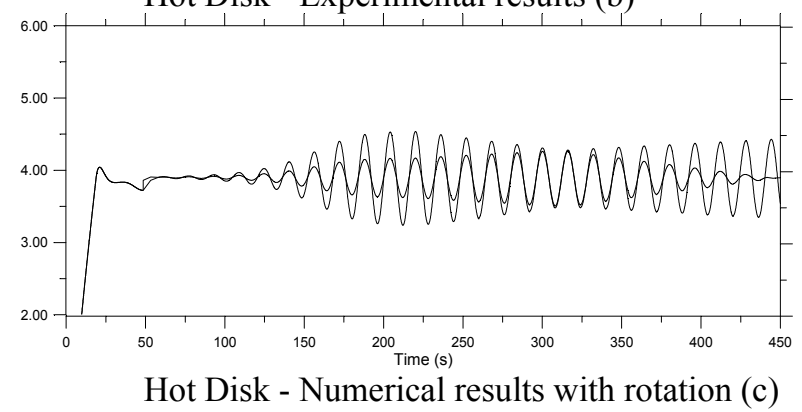

Fig.5 Comparison between numerical and experimental signals of the temperatures measured by two thermocouples near the hot disk shifted at $90^{\circ}$. MET $=$ time $+200 \mathrm{~s}$
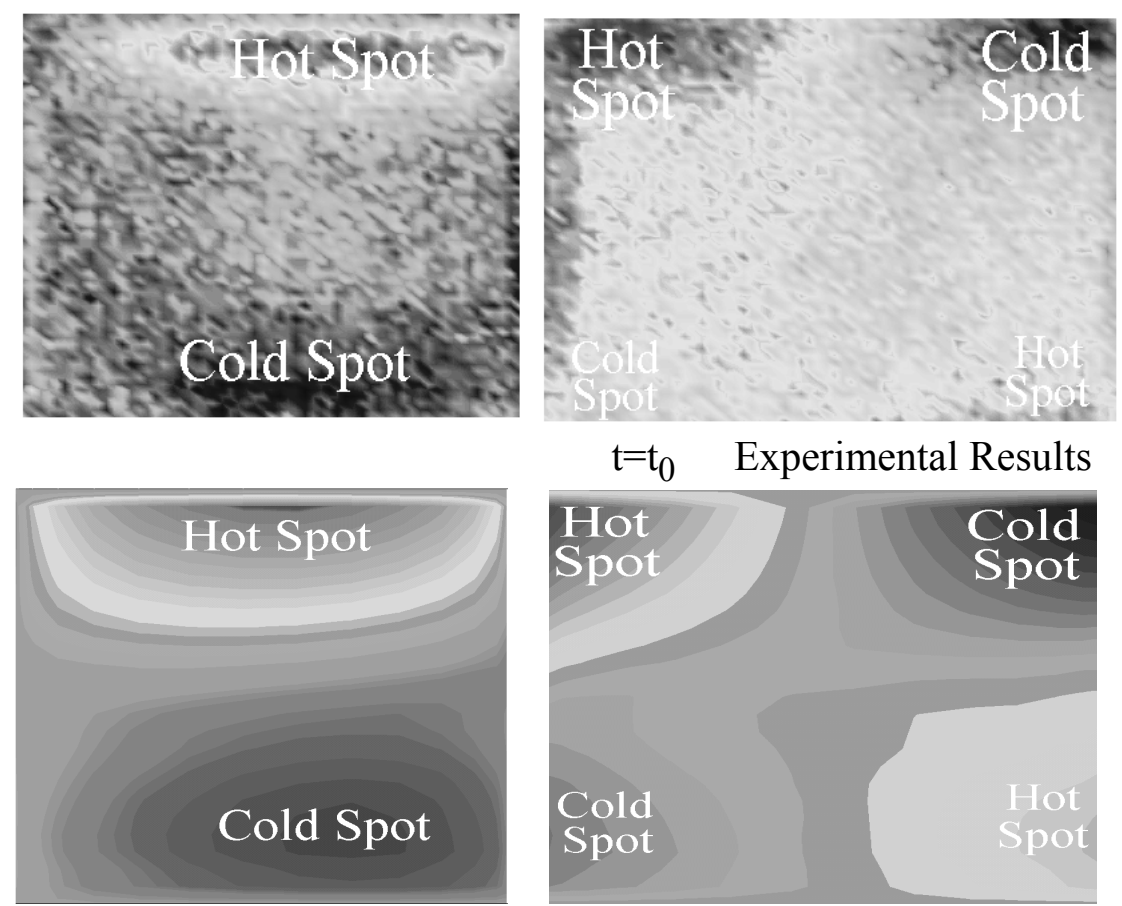
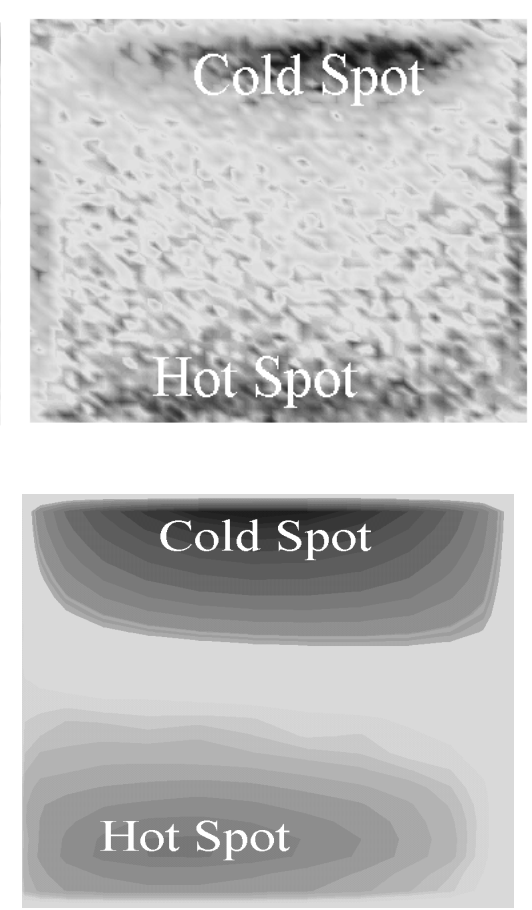

$\mathrm{t}=\mathrm{t}_{0} \quad$ Numerical Results 

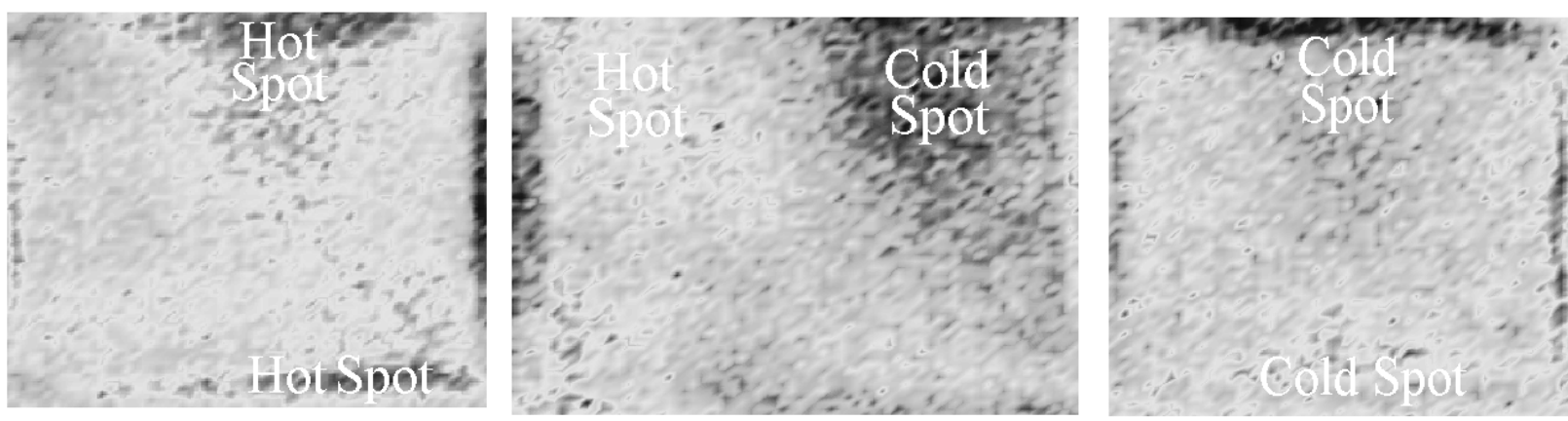

$\mathrm{t}=\mathrm{t}_{0}+\tau / 4$

Experimental Results
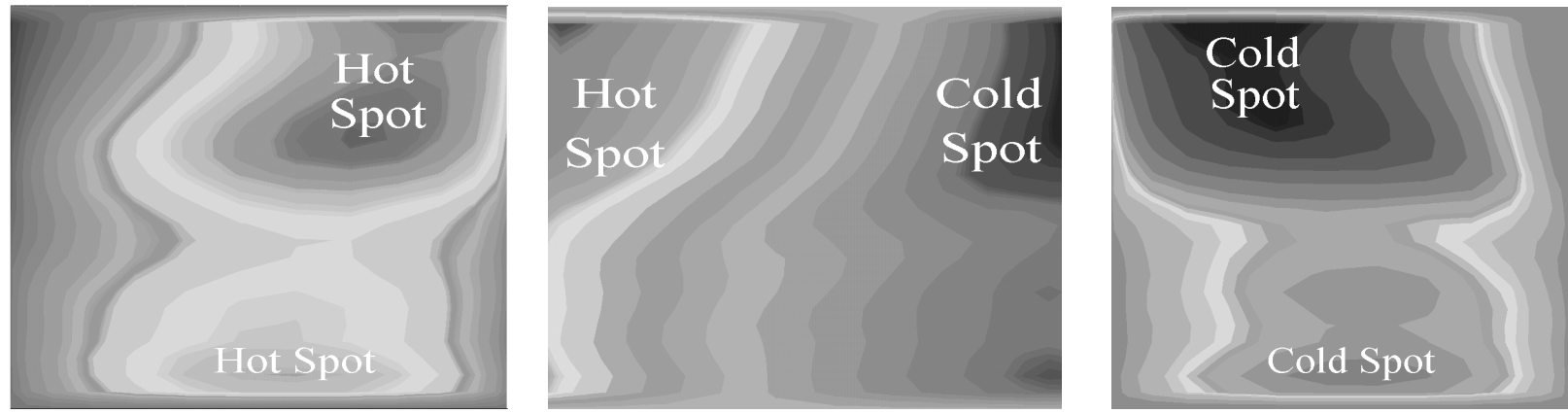

$\mathrm{t}=\mathrm{t}_{0}+\tau / 4 \quad$ Numerical Results
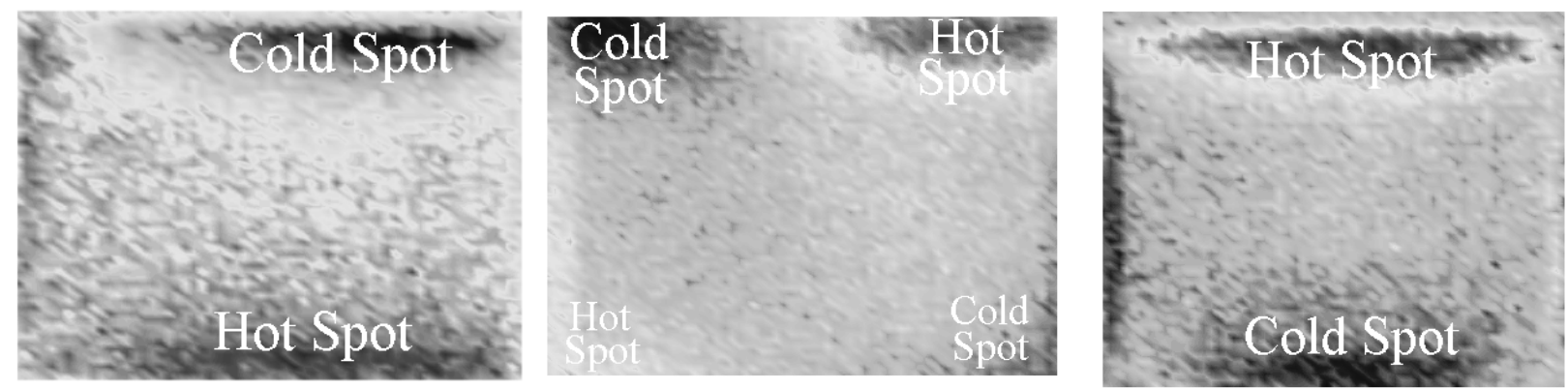

$\mathrm{t}=\mathrm{t}_{0}+\tau / 2 \quad$ Experimental Results
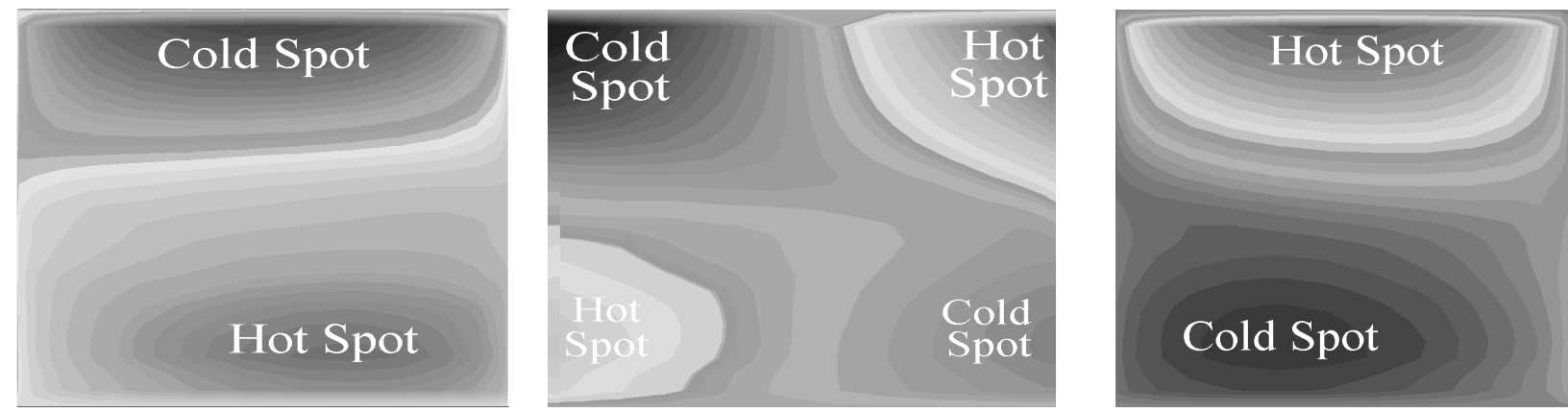

$\mathrm{t}=\mathrm{t}_{0}+\tau / 2 \quad$ Numerical Results

LEFT MIRROR

CENTRAL VIEW

RIGHT MIRROR

Fig.7 Black and white reproduction of the coloured images illustrating the surface temperature disturbances at some moments during one half of the oscillation period $(\tau=13 \mathrm{~s})$ prior to MET $=421 \mathrm{~s}$ 


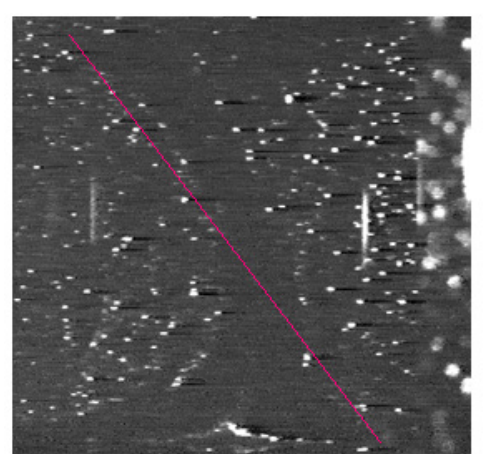

$\mathbf{t}_{\mathbf{0}}$

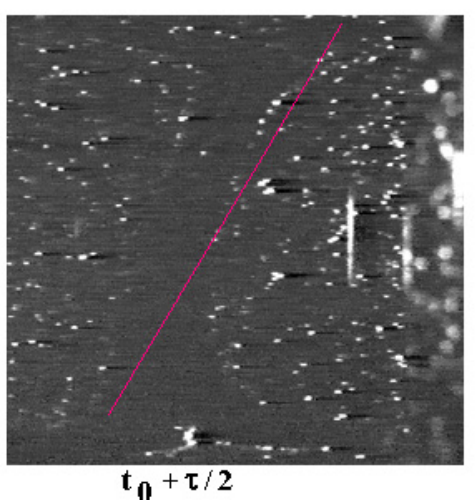

Fig.8 Images of the meridian plane during a period $(\tau=13 \mathrm{~s})$ prior to $\mathrm{MET}=421 \mathrm{~s}$

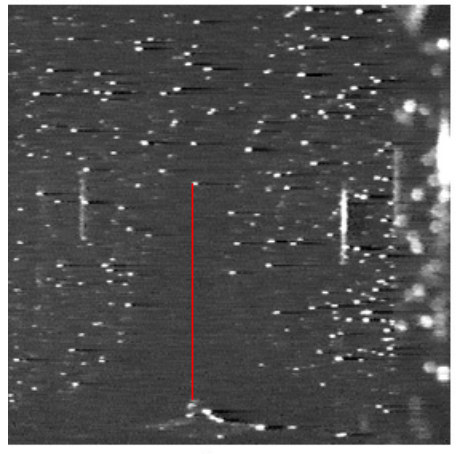

$\mathbf{t}_{\mathbf{0}}$

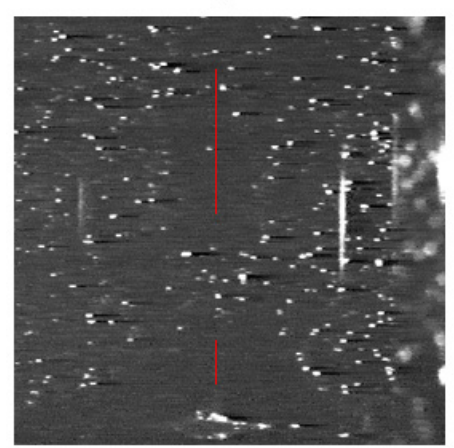

$t_{n}+\tau / 2$

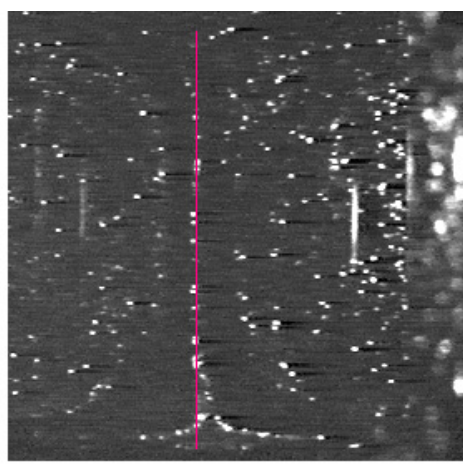

$t_{0}+\tau / 4$

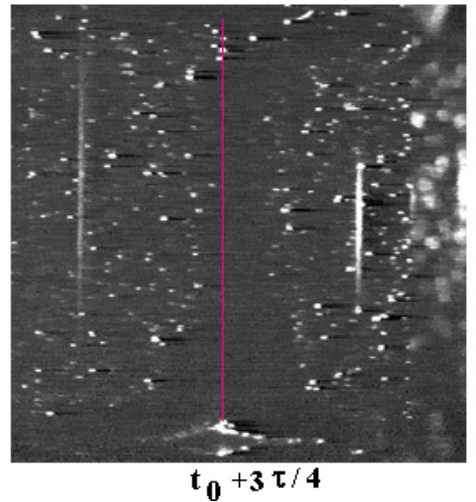

$t_{0}+3 \tau / 4$ 
Finally, Fig8 and Fig.9 show the tracers position in the meridian plane illuminated by the laser beam. The initial time $\mathrm{t}_{0}$ corresponds to $\mathrm{MET}=400 \mathrm{~s}$ in Fig. 8 and to MET $=500 \mathrm{~s}$ in Fig.9 . Each image has been captured 3.2 seconds after the previous one, being the estimated period $\tau \cong 13 \mathrm{~s}$. These images can not identify pulsating or rotating regimes, that are strictly dependent on the azimuthal coordinate, but can only state if the flow is axisymmetric or not. The results are basically in agreement with those obtained by the temperature data. The flow was oscillating in the plane observed by the video camera (pulsation plane) before MET $=421 \mathrm{~s}$. Fig. 8 shows the well known images reported in literature where the motion axis changes direction. On the other hand Fig. 9 shows no inclination of the motion axis, confirming that after MET $=421 \mathrm{~s}$ the motion axis was oscillating in a different pulsation plane.

\section{$\underline{\text { 4. Conclusions }}$}

The analysis of the temperature profiles, measured by thermocouples located near the disks at different azimuthal positions, and the surface temperature distribution, measured by an infrared thermocamera, have confirmed, as anticipated by the numerical simulations, that the oscillatory Marangoni instability appears in the form of a pulsating regime, caused by a hydro-thermal standing wave.

The experimental results seem to indicate that a relative rotation of the pulsating plane around the bridge axis occurred, probably due to the strong angular acceleration caused by the activation of a centrifuge placed near the module TEM 06-4.

This behaviour has been simulated numerically assuming a relative angular speed of the bridge of the order of $0.15 \mathrm{deg} / \mathrm{s}$.

A reflight of the experiment is in preparation during a sounding rocket mission with an undisturbed microgravity environment, to confirm the numerical results already obtained, and to investigate the transition from the pulsating regime to the rotating regime in the available microgravity time.

\section{Acknowledgements}

The authors wish to thank the Italian Space Agency (ASI) for the financial support and the ESA technical staff for the support during the preparation and execution of the experiment.

\section{References}

[1] D. Schwabe, A. Scharmann, F. Preisser, R. Oeder 1978, Experiments on surface tension driven flow in floating zone melting, J. Crystal Growth 43, 305-312

[2] C.H. Chun, W.West, 1979, Experiments on the transition from the steady to the oscillatory Marangoni convection of a floating zone under reduced gravity effect, Acta Astronautica. 6, 1073

[3] R. Monti 1987, On the onset of the oscillatory regimes in Marangoni flows, Acta Astronautica 15, 557-560

[4] F. Preisser, D. Schwabe, A. Scharmann 1983, Steady and oscillatory thermo capillary convection il liquid columns with free cylindrical surface, J.Fluid Mech. 126 , 545567

[5] D. Schwabe, P. Hintz, S. Frank 1996, New Features of Thermocapillary Convection in Floating Zones Revealed by Tracer Particle Accumulation Structures, Microgravity sci. technol. IX/3, 163-168

[6] A. Hirata, M. Sakurai, N. Ohishi, M. Koyama, T. Morita, H. Kawasaki, 1997, Transition process from laminar to oscillatory Marangoni convection in a liquid bridge under normal and microgravity, J.Jpn. Soc. Microgravity Appl 14 (2), 137-143

[7] R. Velten, D. Schwabe, A. Scharmann 1991, The periodic instability of thermocapillary convection in cylindrical liquid bridges, Phys.Fluids A 3, 267-279

[8] S. Frank, D. Schwabe 1998, Temporal and spatial elements of thermocapillary convection in floating zones, Experiments in Fluids, 23, 234-251

[9] Carotenuto L., Castagnolo D., Albanese C. and Monti R., 1998 
Instability of thermocapillary convection in liquid bridges, Phys. Fluids A, 10, 555-565.

[10] R. Monti, R.Savino, M. Lappa, 1998, Influence of geometrical aspect ratio on the oscillatory Marangoni convection in liquid bridges"; 49th IAF Congress, Melbourne, Australia, Sept.28-Oct. 21998

[11] H.C. Kuhlmann, H.J. Rath 1993, Hydrodynamic instabilities in cylindrical thermocapillary liquid bridges, J. Fluid Mechanics,. 247, 247-274

[12] M.Wanshura, V. Shevtsova, H.C. Kuhlmann, H.J.Rath 1995, Convective instability mechanism in thermocapillary liquid bridges, Phys. Fluids A 5, 912

[13] R. Savino and R. Monti, 1996, cylindrical liquid bridges, Phys. Fluids, 8, N. 11,2906

[14] R. Monti, R. Savino, M. Lappa, 1997, Oscillatory Thermocapillary flows in simulated floating zones with time-dependent boundary conditions, Acta Astronautica 41, 863-875.

[15] R. Monti, R. Savino, M. Lappa, 1998, Scientific and technological aspects of a sounding rocket experiment on oscillatory Marangoni flow, Space Forum, 2, 293-318.

[16] M. Lappa and R. Savino, 1999, Parallel solution of the three-dimensional Marangoni flow instabilities in liquid bridges Int. J. Num. Meth. Fluids, 31, issue 5, (August 1999.) 